\title{
PESQUISAS NA ÁREA dE CLASSIFICAÇÃO DE DOENÇAS
}

Ruy Laurenti*

\begin{abstract}
RESUMO:A Classificação Internacional de Doenças (CID) foi adotada internacionalmente em 1893 passando a ser utilizada como uma Classificação de Causas de 77 Morte. A partir da Sexta Revisão, em 1948, a responsabilidade pela CID e sucessivas revisões passou para a OMS e esse instrumento estatístico desde então, vem se ampliando bastante deixando de ser apenas uma classificação de causas de morte para se tornar, a seguir, também, uma classificação de doenças propriamente dita e de motivos de consulta. Atualmente, entende-se pelo jargão CID, uma verdadeira "Família de Classificações". O trabalho descreve, em linhas gerais, pesquisas que são realizadas com a CID, quer como instrumento estatístico de análises de mortalidade e morbidade, quer pesquisas para avaliação ou mesmo para novas propostas quanto aos usos dos múltiplos aspectos englobados sob o título CID.
\end{abstract}

Em 1893 surgiu pela primeira vez um acordo internacional para o uso em todos os países de uma classificação de causas de morte e que passou a ser revista a cada dez anos com finalidade de incorporar novas doenças que iam sendo descritas e que eram causas de morte ${ }^{10}$. Até a quinta revisão, aprovada em 1938 , somente estavam incluidas as doenças que eram causas de morte; a partir da sexta revisão, aprovada em 1948, passou-se a ter uma verdadeira classificação de doenças, visto que não incluía apenas aquelas mortais mas todas as doenças, lesões e mesmo sintomas. Incorporava também alguns motivos de consulta que

\footnotetext{
* Professor Titular do Departamento de Epidemiologia da Faculdade de Saúde Pública da Universidade de São Paulo. Diretor do "Centro Colaborador da OMS Para a Classificação Internacional de Doenças em Português". FSP/USP.
} 
consulta que não eram propriamente doenças 18 A Organização Mundial de Saúde, desde esta sexta revisão, passou a ser a responsável pela chamada "Classificação Internacional de Doenças" e suas sucessivas revisões.

Uma descrição mais detalhada que inclui a parte histórica, a evolução entre as sucessivas revisões, conceitos de classificação e nomenclatura, a estrutura, bem como críticas à classificação podem ser vista na publicação "Análise da Informação em Saúde: 1893-1993, cem anos da Classificação Internacional de Doenças" 10 .

Uma classificação de doenças é um instrumento que agrupa as doenças segundo características comuns e serve, basicamente, para finalidades estatisticas de descrição e análise quanto a distribuição das doenças em uma população definida. É uma sistematização das doenças, sintomas, sinais e motivos de consultas.

\section{PESQUISAS QUE ENVOLVEM A CLASSIFICAÇÃO DE DOENÇAS}

É fácil entender a classificação de doenças como instrumento estatístico para uso em pesquisas. Assim, como exemplo, na descrição e análise da mortalidade por causas ou o mesmo para diagnósticos de internação hospitalar ou assistência ambulatorial, a Classificação Internacional de Doenças é o instrumento utilizado e por todos conhecida.

Nem sempre, porém, fica claro como a classificação de doenças pode ser $\mathrm{o}$ objeto da pesquisa. Entretanto vários tipos de estudos e investigações vêm sendo realizadas, quase que rotineiramente, particularmente na própria área que cuida do assunto na Organização Mundia! da Saúde e em seus escritórios regionais (OPS, EURO, AFRO, etc) sendo que se constitui em atividade quase que 
obrigatória dos dez centros internacionais - "Centros Colaboradores da OMS Para a Classificação de Doenças".

O que se pretende nesta apresentação é mostrar, sem entrar em minúcias, alguns tipos de possibilidades de pesquisas que são realizadas com a classificação internacional de doenças.

Antes, porém, julga-se necessário uma compreensão correta do que atualmente se entende pelo jargão "Classificação Intermacional de Doenças".

No sentido estrito do termo entende-se classificação como um instrumento, como se descreveu antes, que agrupa, segundo uma determinada sistemática, alguma "coisa". No caso essa "coisa" é doença.

Porém, atualmente, sob esse rótulo - Classificação Internacional de Doenças - quase sempre referida ou conhecida pela sua sigla CID, não tem apenas esse sentido estrito, mas, trata-se de um instrumental muito mais amplo. Para se entender o que significa esse "muito mais amplo" é preciso conhecer um pouco da história da CID, que é o que se segue, resumidamente.

No Século XVIII, particularmente durante sua segunda metade, havia um grande interesse na obtenção de um instrumento estatístico que sistematizasse as causas de morte e que fosse de uso internacional principalmente para possibilitar comparações. Após muitas tentativas conseguiu-se, em 1893, um acordo internacional tendo sido proposta e aprovada uma "Classificação de Causas de Morte" que passou a ser usada por vários paises. Aprovou-se, também, uma recomendação para que houvessem revisões periódicas e, no caso, uma periodicidade foi estabelecida como sendo decenal 10 . 
A primeira revisão ocorreu em 1900 e, até a quinta de 1938, era tão somente uma classificação de causas de morte $e$, portanto, continha um reduzido número de códigos. A partir de 1948, com a aprovação da sexta revisão, passou-se a ter uma "classificação de doenças, lesões e causas de morte" e a responsabilidade pelas revisões, como se comentou no inicio, passou para a OMS. Não somente deixou de ser uma classificação de causa de morte mas uma classificação de doenças, no sentido mais amplo, incluindo mesmo aquelas mais banais e possibilitando também classificar motivos de consultas que não eram propriamente doenças. Passou a incluir 0 , então aprovado, certificado médico da causa de morte (atestado de óbito) com as regras para a codificação e classificação das causas de morte, bem como as Listas Especiais para Tabulação de Doenças e os Regulamentos para a compilação e publicação de estatisticas de morbidade e mortalidade 18.

Da Sexta à atual Décima revisão a publicação chamada "Classificação Internacional de Doenças" foi incorporando vários outros tópicos, sendo um deles as definições usadas em estatísticas vitais e de saúde (nascido vivo, perda fetal, períodos e mortalidade infantil, neonatal, perinatal, materna, etc). A própria CID, embora ainda conhecida por essa sigla, passou-se a chamar "Classificação Estatística Internacional de Doenças e Problemas Relacionados à Saúde"14

Atualmente nos órgãos nacionais e internacionais, quer ligados a serviços públicos ou privados de saúde, quer ligados a Universidades, que trabalham com epidemiologia ou tão somente com estatisticas de saúde, o jargão CID engloba numerosos assuntos e não só uma classificação de doenças propriamente dita: mortalidade e morbidade e respectivas regras; definições para apresentações estatisticas onde se inclui as listas especiais, bem como outros itens. Presentemente, a partir da décima revisão, o jargão CID passou também a 
incluir outras classificações, algumas derivadas do "núcleo CID" propriamente dito, sendo que todo o conjunto é chamado "familia de classificações ".

Assim, atualmente, pesquisas em CID englobam toda uma ampla gama de assuntos e/ou temas. E, nesse sentido, é que se passa a descrever alguns tipos de pesquisas sem todavia ter a pretensão de esgotar 0 assunto.

\section{TIPOS DE PESQUISAS COM A CID}

Sem estabelecer uma ordem de importância ou mesmo a quantidade de investigações nos respectivos tipos abaixo enumerados, pode-se descrever os seguintes:

\section{1 - INVESTIGAÇÕES QUE ENVOLVAM O USO DO INSTRUMENTO CLASSIFICAÇÃO DE DOENÇAS PROPRIAMENTE DITA}

Talvez o mais clássico trabalho de pesquisa foi o realizado em 1956 pela Associação Americana de Hospitais e a Associação Americana de Bibliotecárias Médicas. Os hospitais americanos vinham utilizando a Nomenclatura Padrão de Doenças e Operações conhecida pela sigla em inglês SNDO (Standart Nomenclature of Diseases and Operations) ${ }^{1}$ para codificação e, particularmente, para indice diagnósticos de hospitais. Em meados da década de 50 passou a haver interesse da OMS e de muitos hospitais norte-americanos no uso da Classificação Internacional. Aquelas associações programaram e realizaram uma pesquisa comparando ambos instrumentos de trabalho. Participaram catorze hospitais de vários tipos e tamanhos e por um periodo de um ano os prontuários foram codificados segundo as duas maneiras e foi sendo verificado como o indice diagnóstico respondia às solicitações feitas para a retirada de prontuários. $O$ estudo 
demonstrou que a CID se prestava muito bem e com vantagens como instrumento para índice diagnóstico hospitalar 3 .Foi a partir deste estudo, amplamente divulgado, que os hospitais norte americanos foram deixando de usar a SNDO,

substituindo-a pela CID. Atualmente praticamente todos os hospitais no mundo que codificam as causas de internação ou atendimento ambulatorial usam a CID.

A medida que se expandia o uso da CID em hospitais verificou-se que, para muitas especialidades, ela não satisfazia plenamente pois, nesses casos, sempre se desejava mais especificações, isto é, a mesma doença com vários descritores: localizações, manifestações ou outros detalhes. Várias pesquisas em serviço foram realizadas procurando-se adaptações da CID para uso em especialidades médicas e assim surgiram várias como, entre outras, a de oncologia, de estomatologia, de pediatria, de transtornos mentais. Estas atualmente, compõe parte da família de classificações.

Nos últimos anos realizaram-se várias investigações com o objetivo de verificar se a CID poderia ser utilizada em assistência primária de saúde quer quando realizada por médico ou por outro profissional de saúde ou mesmo leigo treinado para tal.

Outras pesquisas poderiam ser citadas e referentes ao uso da CID propriamente dita. É de se destacar numerosas pesquisas do uso da CID em análises de mortalidade por causas múltiplas e, sobre esse tópico alguns comentários serão feitos em outro item. 


\section{2 - PESQUISAS QUE ENVOLVEM AS DEFINIÇÕES ADOTADAS PELA OMS E APRESENTADAS NA CID}

Nesse tipo de pesquisa pode-se considerar aquelas que dizem respeito a verificação quanto ao uso correto de uma determinada definição bem como pesquisas que servem para subsidiar propostas para mudanças de definições ou então ocorrendo uma mudança de definição, investigações para verificar como isso afeta as estatísticas.

Quanto ao uso correto de definições, vários estudos foram realizados mostrando como a não aplicação correta das mesmas pode afetar o nível da mortalidade infantil e da natimortalidade, no caso das definições de nascido vivo e nascido vivo e nascido morto 5

A mortalidade materna é mensurada, segundo a definição, colocando-se no numerador os casos de mortes por complicações da gravidez, do parto e do puerpério, ocorridos até 42 dias após o parto. Várias pesquisas foram realizadas mostrando que, graças aos avanços terapêuticos, freqüentemente ocorrem mortes de mulheres por causas maternas após os 42 dias e a magnitude numérica destas mortes vem aumentando e, portanto, haveria necessidade de estender até um ano o período considerado, isto é, mudar a definição. Várias pesquisas internacionais foram realizadas e mesmo entre nós já foi feito uma pesquisa nesse sentido 8 .

A Décima Revisão da CID 14 mudou a definição do período perinatal o qual passou a começar no inicio da $22^{a}$ semana de gestação e não mais no da $28^{a}$ semana. Assim passa-se a considerar para cálculo da taxa de mortalidade perinatal as perdas fetais a partir de $500 \mathrm{~g}$ o que trará como conseqüência um aumento da taxa e, portanto, afetará os estudos de tendência; e já há estudos mostrando o quanto aumenta 2,11 
Ainda quanto a essa questão de mudança de definição, são necessários estudos mostrando como a legislação dos diferentes paises contempla o registro das perdas fetais, isto é, a partir de quando é obrigatório registrar uma perda fetal. No Brasil, pela legislação vigente, entende-se que devem ser registrados os natimortos, isto é, as perdas fetais a partir da $28^{a}$ semana de gestação. Assim já começaram a ser programadas pesquisas para conhecer como a mudança da definição de periodo perinatal afetará a comparabilidade internacional e se será necessário mudar as legislações vigentes 11.

\section{3 - PESQUISAS SOBRE O USO DAS REGRAS INTERNACIONAIS DE}

\section{CODIFICAÇÃO DE MORTALIDADE}

É bastante conhecida a técnica de avaliação da qualidade da certificação médica da causa de morte por meio da quantificação da proporção de vezes que determinada regra de codificação é aplicada em uma amostra ou no total de atestados de óbitos. Assim, para exemplificar, será tanto melhor a qualidade da certificação, quanto maior a proporção de vezes que se aplica a Regra Geral (a partir da décima revisão passou a chamar-se Principio Geral) o que estaria indicando que o médico declarou as causas segundo uma seqüência lógica. Esses estudos avaliam o uso de todas as regras, quer as de seleção, quer as de modificação da causa básica e são realizados quase que rotineiramente pelos Centros Colaboradores da OMS Para a Classificação de Doenças e por muitos serviços encarregados de produzir as estatísticas de mortalidade no mundo todo 7 .

Outro tipo de pesquisa quanto ao uso das regras para codificar mortalidade já é um pouco mais complexa e exige trabalho de campo. Trata-se de verificar se as "Regras Internacionais Para Codificar Mortalidade", ainda que tidas como "arbitrárias", cumprem bem a função de selecionar a "verdadeira" causa 
básica de morte quando ela é declarada pelo médico de maneira incorreta pela posição ou seqüência no atestado de óbito. Nesse tipo de pesquisa é necessário comparar a causa básica selecionada no atestado de óbito original, isto é, o preenchido pelo médico, com outro atestado refeito preenchido pelo pesquisador seguindo a técnica correta e conhecendo as causas por meio de entrevistas junto aos médicos que cuidaram do caso, consulta a prontuários de hospitais, laudos de autópsias, etc. O "Centro Colaborador da OMS Para a Classificação de Doenças" do Departamento de Epidemiologia da Faculdade de Saúde Pública USP já realizou algumas pesquisas deste tipo 9,17 .

Cada vez mais vem crescendo o interesse e, muitas vezes, até mesmo a necessidade de se analisar a mortalidade segundo aquilo que se convencionou chamar "estatísticas por causa múltipla" ou simplesmente "causas múltiplas de morte". Consiste em tabular as causas de morte segundo todos os diagnósticos informados no atestado de óbito e não somente pela causa básica. A "Investigação Interamericana de Mortalidade na Infância" utilizou essa metodologia para alguns tipos de análises quanto a associação de causas de morte em menores de 5 anos, mostrando, entre outros, a importância da desnutrição em casos nos quais a causa básica era uma doença infecciosa 16 . Com o predomínio das doenças não infecciosas no padrão de mortalidade é importante mostrar a associação existente entre várias doenças ou mesmo mostrar que algumas dessas doenças, como é o caso da hipertensão arterial, que por uma disposição estatistica da CID, não tem prioridade como causa básica de morte em presença de algumas outras doenças, como a doença isquêmica do coração e os vários tipos de acidente vascular cerebral. Assim, como exemplo, no municipio de São Paulo, em adultos de 15 a 74 anos, a hipertensão arterial aparece em 3,0\% dos casos, porém em análises de causas múltiplas ela está presente em $30,0 \%$ dos óbitos, isto é, dez vezes mais 6 . 
A codificação de todas as informações médicas contidas no atestado de óbito com a finalidade de analisar a mortalidade por causas múltiplas requer regras especiais para uso internacional no sentido de que todos trabalham da mesma maneira possibilitando assim comparaçōes. Há algumas propostas de regras mas ainda nenhuma foi oficialmente adotada. Centros Colaboradores da OMS estão, nos últimos anos, realizando pesquisas visando comparar as propostas para se chegar a um acordo internacional.

Quando se compara o padrão de mortalidade entre países e existem taxas que. na comparação, chamam a atenção é usual perguntar: a diferença é real ou é um artefato estatistico? O principal artefato é o uso incorreto das regras de codificação por parte dos codificadores. Um tipo de pesquisa que se realiza freqüentemente é dar a codificadores de dois ou mais paises um mesmo lote de atestado de óbito e comparar a codificação 13,15 .

Algumas vezes o interesse é específico quanto a uma determinada doença sendo assim realizadas pesquisas com atestados de óbito onde ela é informada em qualquer posição; pode-se citar como exemplos casos com diabetes 4 ou com neoplasias 12 .

\section{4 - PESQUISAS NO CAMPO DE APLICAÇÃO DOS DIFERENTES \\ COMPONENTES DA FAMÍLIA DE CLASSIFICAÇÃO}

A CID-10 que foi aprovada pela "Conferência Internacional para a Décima Revisão", em 1989, e aprovada pela "Quadragésima Terceira Assembléia Mundial da Saúde" incorporou o conceito de uma "família de classificações", o que vinha sendo discutido e trabalhado desde a Nona Revisão. Na CID-10 explicita-se o seguinte:... "Durante a preparação da Nona Revisão percebeu-se que a CID, por si 
só, não poderia abranger todas as informações necessárias e que apenas uma "familia" de classificações sobre doenças e outros problemas relacionados à saúde poderia suprir as várias necessidades em saúde pública. Desde o final da década de 70 , várias soluções possiveis têm sido consideradas, uma das quais demandava uma classificação central (CID) com uma série de módulos, alguns hierarquicamente relacionados e outros de caráter suplementar"14.

Foi após vários estudos e discussões em cooperação com os Centros Colaboradores que se elaborou um conceito de familia de classificação e que foi incorporado na CID-10.

A CID tradicional (nivel de três caracteres) é o núcleo central da família e o esquema abaixo é auto explicativo para se entender o conceito da família de classificações. 
Familia das classificaçōes de doenças e de problemas relacionados à saúde

INFORMAÇÁO DE APOIO PARA ASSLSTÉNCL MÉOICA

- Inlormacoes de saide por pessoed niso midico (Lay reporting)

- Ovisos lipos de Intormecóes de savide basesdoes ne comunidade
OUTRAS ClASSUFICAÇȮES RELACIONAOAS À sAUjoe

- Deficibriclas. Incapacidadese Deaventagena

- Procedimentos

- Motivos e consulla (queiras)

\section{CLASSIFICAÇẢO ESTATISTICA INTERNACIONAL DE DOENÇAS \\ E DE PROBLEMAS \\ RELACIONADOS A SAUDDE}

CLASSIFICAÇAO NU-

CLEAR de 3 CARACTERES

- Diagnóstico

- Sintomas

- Achados enormals de laboratólo

- Lesdes a envenenamentos

- Causas externas de morbidade e mortalidade

- Falores que influenciem o estado de soude

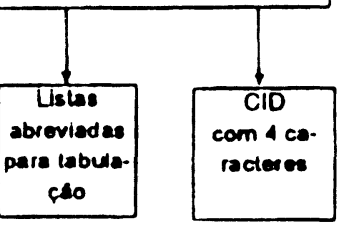

ADAPTAÇÓES PARA ESPECLALIDADES

- Oncologia

- Odontotogla e estomatotogia

- Dermatotogia

- Psiquidatria

- Nevorotogia

- Obeleticicia e ginecologra

- Reumalologia * orropedia

- Podiatria, elc.
MOMENCLATUAA INTERNACIONAL DE DOENÇAS 
Pelo esquema verifica-se a existência das várias outras classificações membros da familia, tais como: as adaptações para especialidades médicas; para informações em serviços de atenção primária à saúde; para deficiências, incapacidades e desvantagens; para procedimentos em medicina e outros. $\dot{E}$ desejável também, como membro da família, a existência de uma "Nomenclatura Internacional de Doenças" cuja finalidade é prover um termo único para cada doença.

Do exposto acima deduz-se facilmente as possibilidades e mesmo necessidade de estudos e pesquisas que envolvam os vários membros da família por si e, muito importante, no relacionamento e mesmo superposição deles entre si. De fato, várias pesquisas nesse sentido vêm sendo realizadas, destacando-se aquelas referentes a uso de uma "Classificação de Procedimentos em Medicina", "Classificação de Deficiências, Incapacidades e Desvantagens" e a "Classificação de Informações em Atenção Primária à Saúde". Nos próximos anos tudo leva a crer que as investigações nesses assuntos especificos representarão grande parte das atividades daqueles que vêm trabalhando no, atualmente, extenso campo da CID, "senso-lato".

\section{REFERÊNCIAS BIBLIOGRÁFICAS}

1. AMERICAN MEDICAL ASSOCIATION - Standart nomenclature of diseases and operations. 4 ed., Blackistan Company, 1952.

2. BLOUDEL, B. \& BRÉART, G. Mortinatalité et mortalité néonatalle: description, facteurs de risque et evaluation des soins. Encycl. Méd. Chir. (Paris). Obstétrique 5-077-C-20. Pediatrice 4-002-F-50. 1994.6p.

3. EFFICIENCY in hospital indexing of the coding system of the International Statistical Classification and Standart Nomenclature of Diseases and Operations. J.Am. Ass.Med. Rec. Libr., 30(3)1959. 
4. JOUGLA, E. Comparison of practice in coding diabetes or a cause of death in eight European Countries - Results of the "EURODIAB" concerted Eumpean activity. São Paulo, 1991. (WHO - SES/ICD/C/ 91.10).

5. LAURENTI, R. Fatores de erro na mensuração da mortalidade infantil. Rev. Saúde Pública, 9: 529-37, 1975.

6. LAURENTI, R. Epidemiologia da hipertensão arterial. In: Chiaverini, R. et al. Doença hipertensiva: diagnóstico, etiopatogênese, tratamento. São Paulo, Livraria Atheneu, 1980.

7. LAURENTI, R. The use of coding rules for underlying cause of death. Budapest, Hungary, 1983. (WHO - DESMG/83.5).

8. LAURENTI, R. et al. Mortalidade de mulheres em idade fértil no município de São Paulo (Brasil), 1986. II - Mortes Por Causas Maternas. Rev. Saúde Pública, 19: 225-32, 1990.

9. LAURENTI, R. Accuracy of causes of death statements on draft certificates. São Paulo, Brazil. (WHO - SES/ICD/C/9I.5).

10. LAURENTI, R. Análise da informação em saúde: 1893-1993, cem anos da Classificação Internacional de Doenças. Rev. Saúde Pública, 25:407-17, 1991.

11. LAURENTI, R. Período perinatal e mortalidade perinatal: conceitos e definições. São Paulo, 1994 [Preparado para a área materno-infatil da Organização Panamericana da Saúde].

12. LAURENTI, R. et al. Death certificates with mention of neoplasm: comparison of the selection and codification of the underlying cause of death between coders from Tokio (Japan) and São Paulo (Brazil). Report of a pilot study, Part I. Beijing, China, 1992. (WHO - SES/ICD/C/92.5).

13. LOBO DA COSTA JR., M. \& FONSECA, L. A. Comparison between the selection of underlying cause of death by the North American and Brazilian Centres: results of the codification of North American death certificates by the Brazilian Centre. San Francisco, 1984. (WHO - DES/ICD/C/84.42).

14. ORGANIZAÇÃO MUNDIAL DE SAÚDE - Classificação estatística internacional de doenças e problemas relacionados a saúde. $10^{a}$ rev., 1989 , São Paulo, EDUSP, 1993. 
15. PERCY, C. International death certificates study: comparison of coding underlying cause of death by ICD-9 in different countries - São Paulo, Brazil, 1985. (WHO; DES/ICD/C/85.18).

16. PUFFER, R. R. \& SERRANO, C. V. Patterns of mortality in childhood. Washington, D. C. Pan American Health Organization, 1973. (PAHO Scient. Public. $\left.n^{\circ} 267\right)$.

17. SANTO, A. H. et al. Evaluation of the uses of the rules for selection of causes of death for primary mortality statistics. Meknes, Marocco, 198I. (WHO ICD/81.1)4.

18. WORLD HEALTH ORGANIZATION - Manual of the international statiscal classification of diseases, injuries, and causes of death. 6th rev., 1948. Geneva, Switzerland, 1948. 